\title{
An ANKRD11 exonic deletion accompanied by a congenital megacolon in an infant with KBG syndrome
}

\author{
Go Hun Seo', Arum Oh', Minji Kang², Eun Na Kim³, Ja-Hyun Jang', Dae Yeon Kim5, Kyung Mo Kim, Han-Wook Yoo ${ }^{1,6}$, and \\ Beom Hee Lee ${ }^{1,6, *}$ \\ ${ }^{1}$ Department of Pediatrics, Asan Medical Center, University of Ulsan College of Medicine, Seoul, Korea \\ ${ }^{2}$ Asan Institute for Life Sciences, University of Ulsan College of Medicine, Seoul, Korea \\ ${ }^{3}$ Department of Pathology, Asan Medical Center, University of Ulsan College of Medicine, Seoul, Korea \\ ${ }^{4}$ Genome Research Center, Green Cross Genome, Yongin, Korea, \\ ${ }^{5}$ Department of Pediatric Surgery, Asan Medical Center, University of Ulsan College of Medicine, Seoul, Korea \\ ${ }^{6}$ Medical Genetics Center, Asan Medical Center, University of Ulsan College of Medicine, Seoul, Korea
}

KBG syndrome is an autosomal dominant syndrome presenting with macrodontia, distinctive facial features, skeletal anomalies, and neurological problems caused by mutations in the ankyrin repeat domain 11 (ANKRD11) gene. The diagnosis of KBG is difficult in very young infants as the characteristic macrodontia and typical facial features are not obvious. The youngest patient diagnosed to date was almost one year of age. We here describe a 2-month-old Korean boy with distinctive craniofacial features but without any evidence of macrodontia due to his very early age. He also had a congenital megacolon without ganglion cells in the rectum. A de novo deletion of exons 5-9 of the ANKRD11 gene was identified in this patient by exome sequencing and real-time genomic polymerase chain reaction. As ANKRD11 is involved in the development of myenteric plexus, a bowel movement disorder including a congenital megacolon is not surprising in a patient with KBG syndrome and has possibly been overlooked in past cases.

Key words: KBG syndrome, ANKRD11.

\section{Introduction}

KBG syndrome (OMIM \# 148050) was first described in 1975 as an autosomal dominant disorder associated with characteristic macrodontia, distinctive facial features, skeletal anomalies, and neurological problems [1]. KBG represents the surname initials of the first families diagnosed with the disorder. The diagnostic criteria for KBG syndrome were proposed in 2007. That is, a preliminary diagnosis of this disorder could be made when a patient had four or more of the following seven clinical features: macrodontia of the upper central incisors, distinctive facial features, hand anomalies, neurological involvement with global developmental delay, delayed bone age, costovertebral anomalies, postnatal short stature, and a first degree relative with KBG syndrome [2].

ANKRD11, encoding ankyrin repeat domain-containing pro-

Received: 1 October 2018, Revised: 10 December 2018, Accepted: 12 December 2018, Published: 30 June 2019

${ }^{*}$ Corresponding author: Beom Hee Lee, M.D., Ph.D. (iD https://orcid.org/0000-0001-9709-2631

Department of Pediatrics, Asan Medical Center, University of Ulsan College of Medicine, 88 Olympic-ro 43-gil, Songpa-gu, Seoul 05505, Korea.

Tel: +82-2-3010-5950, Fax: +82-2-473-3725, E-mail: bhlee@amc.seoul.kr

Conflict of interest: The authors declare that they do not have any conflicts of interest.

(ㄷ) This is an open-access article distributed under the terms of the Creative Commons Attribution Non-Commercial License (http://creativecommons.org/licenses/by-nc/4.0/) which permits unrestricted non-commercial use, distribution, and reproduction in any medium, provided the original work is properly cited.

(c) Copyright 2019 by the Korean Society of Medical Genetics and Genomics 
tein 11, was identified as the gene responsible for KBG syndrome in 2011 [3]. Previous molecular testing of ANKRD11 helped to identify additional patients with KBG syndrome in whom the clinical phenotypes did not fulfill the previously-proposed diagnostic criteria $[4,5]$. Moreover, additional but uncommon phenotypes have now been associated with this condition such as cryptorchidism, conductive hearing loss, congenital heart defects, and palatal defects [4]. The diagnosis of KBG syndrome is difficult in very young infants as the characteristic macrodontia and typical facial features are not obvious. The youngest patient diagnosed to date was almost one year old $[5,6]$.

We here describe a two-month-old infant who presented with dysmorphic facial features and multiple anomalies. KBG syndrome was suspected due to a large exonic deletion of $A N$ $K R D 11$ gene detected by exome sequencing and confirmed by genomic quantitative polymerase chain reaction (PCR). Of note, this patient had a congenital megacolon which has not been described previously with this syndrome.

\section{Case}

The study case was the third child of non-consanguineous Korean parents. He was delivered by cesarean section at 37 gestational weeks with a birth weight of 2,580 $\mathrm{g}$ (standard deviation [SD] score, -1.0$)$ and height of $47 \mathrm{~cm}(-1.1 \mathrm{SD}$ score). The pregnancy, labor, and delivery were uneventful. Immediately after birth, however, he was admitted to the neonatal intensive care unit due to transient tachypnea of newborn and feeding intolerance. Abdominal ultrasonography revealed diffuse luminal dilatation of the entire colonic loop with fecal material, but he passed meconium during the first 1 to 2 days after birth. He showed distinctive craniofacial features, including a coarse face, frontal bossing, mild synophrys, anteverted nares, a thin upper lip with marked cupids bow, a high-arched palate, and bifid uvula. Right cryptorchidism, single transverse palmar crease, and left foot polydactyly were also evident. Echocardiography revealed a small atrial septal defect and patent duct arteriosus. His karyotype was 46, XY and a chromosomal microarray was normal. His symptoms improved after supportive care and he was discharged at 20 days after birth.

Following discharge, the patient had no stool passage for 10 days and showed abdominal distension. A colon study performed at 1 month of age revealed a markedly dilated colon and transition zone in the proximal part of sigmoid colon, which indicated a congenital megacolon (Fig. 1A). The congenital megacolon was then surgically corrected with a laparoscopic endorectal pull-through operation at 1 month of age. The pathological findings indicated an absence of ganglion cells in the rectum (Fig. 1B-1) but these cells were observed in the sigmoid colon (Fig. 1B-2). Immunostaining of Bcl-2 and calretinin, which are biomarkers of enteric ganglion cells, was negative in the distal part of the sigmoid (Figs. 1C-1 and 2), suggesting the existence of a transitional zone.

We decided to perform targeted exome sequencing due to difficulty of suspicion of specific disease, despite clinical information and dysmorphic facial features. Following written
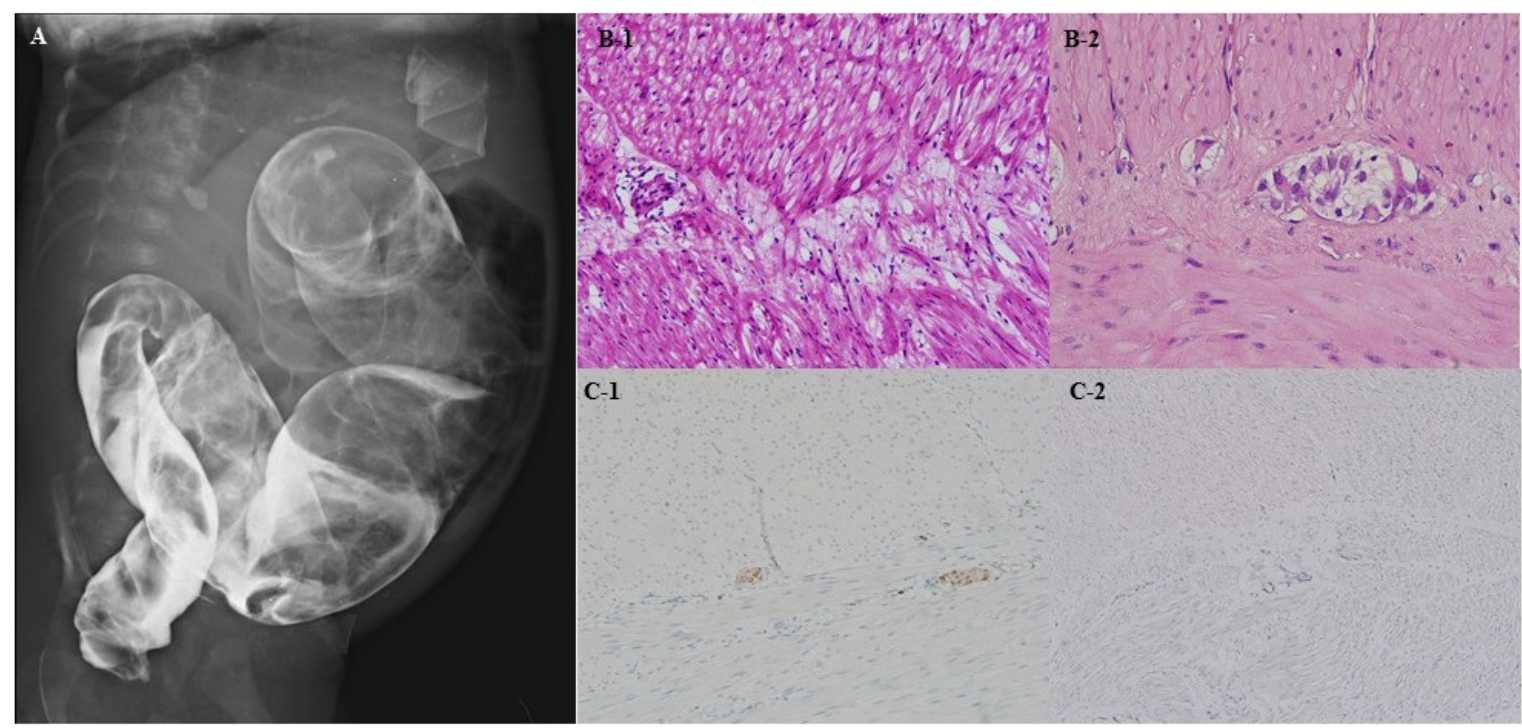

Fig. 1. (A) A colon study of the patient revealed a dilated colon above the transition zone in the proximal part of sigmoid colon. (B-1) Absence of ganglion cells in the rectum (H\&E stain, $\times 200)$. (B-2) Detection of ganglion cells in the sigmoid colon (H\&E stain, $\times 200) .(C) B c l-2(C-1, \times 100)$ and calretinin immunostaining $(C-2, \times 100)$ was negative in the distal part of the sigmoid. 
informed consent, blood samples were collected for exome sequencing from the parents and the patient at 2 months of age using genomic DNA (gDNA) from peripheral blood leukocytes. Exomes were captured using the TruSight One Panel (Illumina Inc., San Diego, CA, USA), which enriches a 12-Mb region spanning 4,813 genes. Sequencing was then performed on a Next Seq platform (IIlumina Inc.) and the sequence reads were aligned to the reference genome, hg19, using a Burrow-Wheeler Aligner (version 0.7.12; MEM algorithm). The mean depth of coverage was $90 \times(\%$ of $>10 \times=98 \%)$. Real time-polymerase chain reaction (RT-PCR) was performed to assess the gDNA content of the ANKRD11 gene (NM_013275.5) in the patient, parents, and in a control. Total gDNA was extracted from peripheral blood lymphocytes using the Gentra Puregene kit (Qiagen Inc., Valencia, CA, USA). The PCR reactions were carried out in a total volume of $10 \mu \mathrm{L}$, containing $50 \mathrm{ng}$ of gDNA template, 10 pmol of each primer, and SYBR Premix Ex Taq (Takara Bio Inc., Shiga, Japan). The comparative threshold cycle method was used to determine the relative quantification of gDNA expression levels. A deletion was suspected in exons 5-9 of the ANKRD11 gene following targeted exome sequencing. RT-PCR analysis of ANKRD11 gene

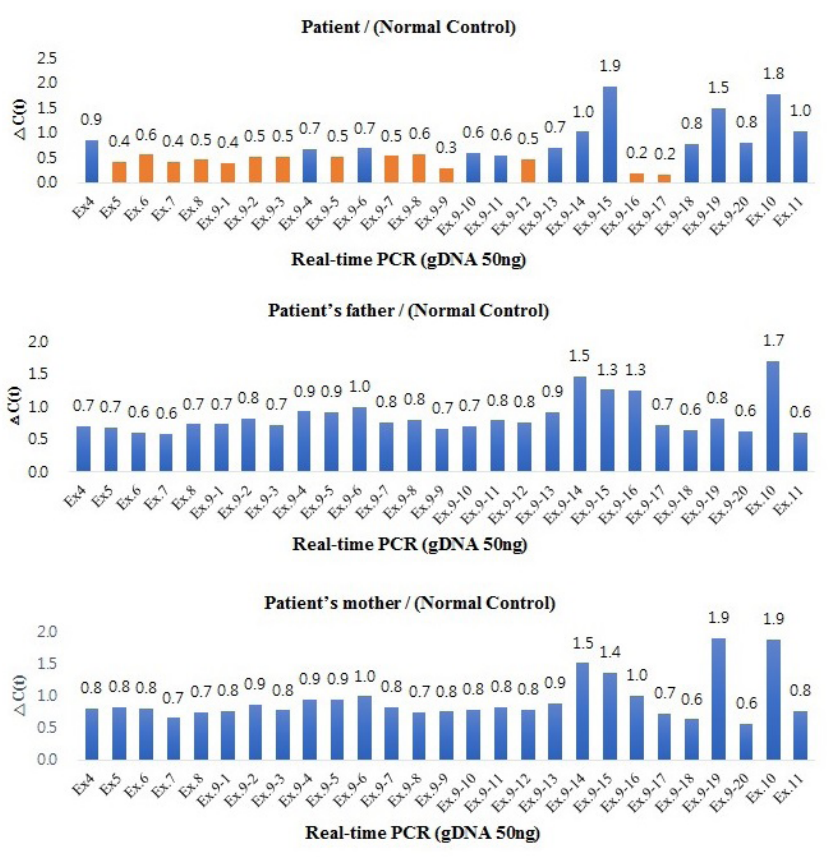

Fig. 2. Results of real-time polymerase chain reaction (PCR) to assess the genomic DNA (gDNA) content of the ANKRD11 gene in the study patient and his parents compared to a normal control. A deletion of exons 5-9 in the patient indicated gene dosage ratios of 0.2 to 0.6 . The parents did not have these deletions and showed ratios of 0.7 to 1.9. The gDNA for the exon 5-9 of ANKRD11 gene expression is less abundant in the patient than in the normal control. expression suggested a haploinsufficiency of exons 5-9 in the patient compared to the normal control (Fig. 2). Following the combined exome sequencing and RT-PCR results, the patient was diagnosed with KBG syndrome caused by a deletion of exons 5-9 in the ANKRD11 gene.

At 2 months of age, the patient had feeding problems such as aspiration, and respiratory difficulties due to a velopharyngeal insufficiency and weak sucking power. Tube feeding was therefore commenced. His right cryptorchidism was surgically corrected at 9 months of age. He could control his head and roll over at 11 months of age. At the latest evaluation at 1 year, his height was $77.4 \mathrm{~cm}$ (0.5 SD score), and body weight was $9.4 \mathrm{~kg}$ ( $-0.5 \mathrm{SD}$ score). He had no vertebral and rib anomalies on X-ray and physical examination.

\section{Discussion}

To date, about 100 cases of KBG syndrome due to an $A N$ $K R D 11$ haploinsufficiency have been reported $[4,5,7,8]$. Most of these patients have shared common clinical features, such as macrodontia, variable degrees of developmental delay, short stature, hand anomalies, and distinctive facial features, including a prominent nasal bridge with bulbous nasal tip, long and smooth philtrum, thin upper lip, and brachycephaly with a triangular face $[4,5]$. Additional features that have been reported include seizures, cardiac abnormalities (15-25\%), conductive hearing loss (32\%), palatal abnormalities (10-20\%), and velopharyngeal insufficiency $[4,5]$. However, with the further development of genetic diagnostic techniques, more KBG syndrome cases without the full spectrum of clinical features have been identified such as our current case.

Most of the reported ANKRD11 mutations in KBG syndrome patients have been frameshift and nonsense, the majority of which have been located in exons 9 and $10[4,5,8]$. Differently sized exonic ANKRD11 deletions have also been reported in 6 patients. However, including our present case, and there appears to be no differences in phenotypic severity according to deletion size [5].

ANKRD11 mutations are associated with developmental delay and autistic spectrum disorder as this gene is expressed in the brain and its deficiency primarily affects neuronal and glial cells [3]. The ANKRD11 protein is also involved in chromatin remodeling for histone acetylation and gene expression in neural precursor cells. In addition, ANKRD11 has recently been suggested to induce dendrite growth and migration in cortical neurons through the brain-derived neurotrophic factor (BDNF) 
and its receptor, tropomyosin-related kinase B (TrkB) signaling [9]. Hence, its deficiency reduces the proliferation of neural precursors and neurogenesis, and leads to aberrant neuronal positioning [10]. Of note also, BDNF and TrkB are also expressed in the myenteric plexus and are involved in enhancing enteric nervous system signaling and gastrointestinal motility $[11,12]$. In an aganglionic intestine, BDNF and TrkB expression is diminished [13]. Therefore, an ANKRD11 deficiency is quite probably associated with a congenital megacolon via the reduced BDNF/ TrkB signaling in KBG syndrome. Gastrointestinal involvement with $\mathrm{KBG}$ syndrome such as gastro-esophageal reflux disease and feeding difficulty has been reported $[5,8]$. In addition, a recent Austrian study reported a case of KBG syndrome with an abdominal viscera and vessel malrotation [14]. However, bowel movement disorders including a congenital megacolon had not been reported with this condition until our present case study.

In conclusion, our current case report expands the clinical spectrum of KBG syndrome. Considering the relationship between ANKRD11 and BDNF/TrkB signaling, an evaluation of bowel movement disorders is necessary in future assessments of this condition.

\section{Acknowledgements}

This study was supported by a grant from the National Research Foundation of Korea, funded by the Ministry of Education, Science, and Technology (NRF-2018M3A9H1078335).

\section{References}

1. Herrmann J, Pallister PD, Tiddy W, Opitz JM. The KBG syndrome-a syndrome of short stature, characteristic facies, mental retardation, macrodontia and skeletal anomalies. Birth Defects Orig Artic Ser 1975;11:7-18.

2. Skjei KL, Martin MM, Slavotinek AM. KBG syndrome: report of twins, neurological characteristics, and delineation of diagnostic criteria. Am J Med Genet A 2007;143A:292-300.

3. Sirmaci $A$, Spiliopoulos $M$, Brancati $F$, Powell $E$, Duman D, Abrams $A$, et al. Mutations in ANKRD11 cause KBG syndrome, characterized by intellectual disability, skeletal malformations, and macrodontia. Am J
Hum Genet 2011;89:289-94.

4. Ockeloen $\mathrm{CW}$, Willemsen $\mathrm{MH}$, de Munnik $\mathrm{S}$, van Bon BW, de Leeuw $\mathrm{N}$, Verrips $A$, et al. Further delineation of the KBG syndrome phenotype caused by ANKRD11 aberrations. Eur J Hum Genet 2015;23:1176-85.

5. Goldenberg A, Riccardi F, Tessier A, Pfundt R, Busa T, Cacciagli P, et al. Clinical and molecular findings in 39 patients with KBG syndrome caused by deletion or mutation of ANKRD11. Am J Med Genet A 2016;170:2847-59.

6. Kim HJ, Cho E, Park JB, Im WY, Kim HJ. A Korean family with KBG syndrome identified by ANKRD11 mutation, and phenotypic comparison of ANKRD11 mutation and 16q24.3 microdeletion. Eur J Med Genet 2015;58:86-94.

7. Miyatake $S$, Okamoto $N$, Stark Z, Nabetani M, Tsurusaki Y, Nakashima $M$, et al. ANKRD11 variants cause variable clinical features associated with KBG syndrome and Coffin-Siris-like syndrome. J Hum Genet 2017;62:741-6.

8. Low K, Ashraf T, Canham N, Clayton-Smith J, Deshpande C, Donaldson $A$, et al. Clinical and genetic aspects of KBG syndrome. Am J Med Genet A 2016;170:2835-46.

9. Ka M, Kim WY. ANKRD11 associated with intellectual disability and autism regulates dendrite differentiation via the BDNF/TrkB signaling pathway. Neurobiol Dis 2018;111:138-52.

10. Gallagher D, Voronova A, Zander MA, Cancino GI, Bramall A, Krause MP, et al. Ankrd11 is a chromatin regulator involved in autism that is essential for neural development. Dev Cell 2015;32:31-42.

11. Weese-Mayer DE, Bolk S, Silvestri JM, Chakravarti A. Idiopathic congenital central hypoventilation syndrome: evaluation of brainderived neurotrophic factor genomic DNA sequence variation. Am J Med Genet 2002;107:306-10.

12. Hoehner JC, Wester T, Påhlman S, Olsen L. Alterations in neurotrophin and neurotrophin-receptor localization in Hirschsprung's disease. J Pediatr Surg 1996;31:1524-9.

13. Boesmans W, Gomes P, Janssens J, Tack J, Vanden Berghe P. Brainderived neurotrophic factor amplifies neurotransmitter responses and promotes synaptic communication in the enteric nervous system. Gut 2008;57:314-22.

14. Murray N, Burgess B, Hay R, Colley A, Rajagopalan S, McGaughran J, et al. KBG syndrome: an Australian experience. Am J Med Genet A 2017;173:1866-77. 